\title{
The Research on Topological Reconstruction Method of Triangular Mesh under Multi-core Environment
}

\author{
Huijiang Zheng ${ }^{1,3, a}$, Jing Zhang ${ }^{2, b}$, Ying $\operatorname{Tian}^{1, c}$ and Jianming Che ${ }^{1, d}$ \\ ${ }^{1}$ Tianjin Key Lab. for Advanced Manufacturing Tech. \& Equipment, Tianjin University, Tianjin 300072, \\ China \\ ${ }^{2}$ School of Control \& Mechanical Engineering, Tianjin Chengjian University, Tianjin 300384, China \\ ${ }^{3}$ School of Mechanical \& Aerospace Engineering, Queen's University Belfast, Belfast BT9 5AH, UK. \\ atom_zhj@126.com, bemmyzj@163.coml, ctianying@tju.edu.cn, ${ }^{d}$ tcjm@tju.edu.cn
}

Keywords: multi-core environment, triangular mesh, topological reconstruction.

\begin{abstract}
Topological reconstruction for Triangular mesh model was the preconditions for discrete data to be widely applied in modern manufacturing. Using Hash table as auxiliary data structure, transform half edge matching structure based on the comparison of marked values floating point numbers to integer matching structure based on index value, and puts forward the topological reconstruction method of triangular mesh under multi-core environment. This method played the characteristics of multi-core environment, effectively improve the efficiency of processing hash function conflict and reduce the concurrent access conflict. Filter the redundant data of triangular mesh, at the same time complete its internal topological relation reconstruction. The calculation examples demonstrate the efficiency and effectiveness of the present method.
\end{abstract}

\section{Introduction}

Multi-core processors integrate with multiple single thread processing cores or multiple simultaneous multi thread processor cores in the same processor. The whole processor chip can realize the parallel execution of multiple threads on physics. It will improve the efficiency of the processor for processing data [1, 2].

Because the triangular mesh data has the characteristics of simple format, easy to read [3]. And because by the triangulation can express and transfer all kinds of complex surface parts surface information, it has become one of the more commonly used model data in the manufacturing technology field [4]. But the triangular mesh file only lists the vector and the coordinate values of the vertices of triangular faces which are obtained after the triangulation of part surface, and does not contain the basic topological relations between various geometric elements in the triangular mesh and constitute information of part surfaces [5]. In addition, the vertex coordinates information in the triangular mesh file is recorded repeatedly in multiple triangular faces. So the data redundancy phenomenon is serious. In modern manufacturing, part surfaces structure is more and more complex, the high accurate triangulation results lead to produce a large amount of triangular mesh model. Due to the triangular mesh model has above shortcomings, which bring great difficulties to analyze and calculate to part surfaces. Therefore, need incorporate a large number of redundant data in a triangular mesh, set up no duplicate data surface, the vertex table and side table, and reconstruct accurately topology information between various geometric elements.

If use direct traversal method to query and merge a large number of redundant data, the efficiency is very low $[5,6]$. In order to improve the efficiency of filtering redundancy data in the triangular mesh model and topological information reconstruction, many domestic and foreign scholars have done a lot of research [5-7]. In reconstructing boundary topological information, the existing methods mainly use the half edge matching $[5,6]$. It need match exactly and approximately by float to boundary endpoint coordinates. The implementation process is complex. Because the existing methods have research all under the single-core environment, the calculation efficiency is decreased obviously to process large amount of data. 
In this paper, using multi-core environment, transform floating point matching based on coordinates to integer matching based on index value. With the Hash table structure for auxiliary data structure, through reducing the probability of concurrent access conflicts, put forward the topological reconstruction method of triangular mesh under multi-core environment. This method can effectively improve the computation efficiency of topological reconstruction of triangular mesh.

\section{Topological reconstruction of triangular mesh}

Build the structure of parallel-hashing. If establish a $\mathrm{f}$ rule between the triangular coordinate values and the array index values, such that

$$
f: \boldsymbol{R}^{3} \rightarrow \boldsymbol{N}
$$

There is and satisfy $\forall \boldsymbol{A}_{1}\left(a_{1}, b_{1}, c_{1}\right), \boldsymbol{A}_{2}\left(a_{2}, b_{2}, c_{2}\right) \in \boldsymbol{R}^{3}$, if $\boldsymbol{A}_{1} \neq \boldsymbol{A}_{2}$, then $f\left(\boldsymbol{A}_{1}\right) \neq f\left(\boldsymbol{A}_{2}\right)$ and the $f$ rule known as the Hash function[8]. To query by Hash function, need to calculate objects images only once, can judge whether the object already exists.

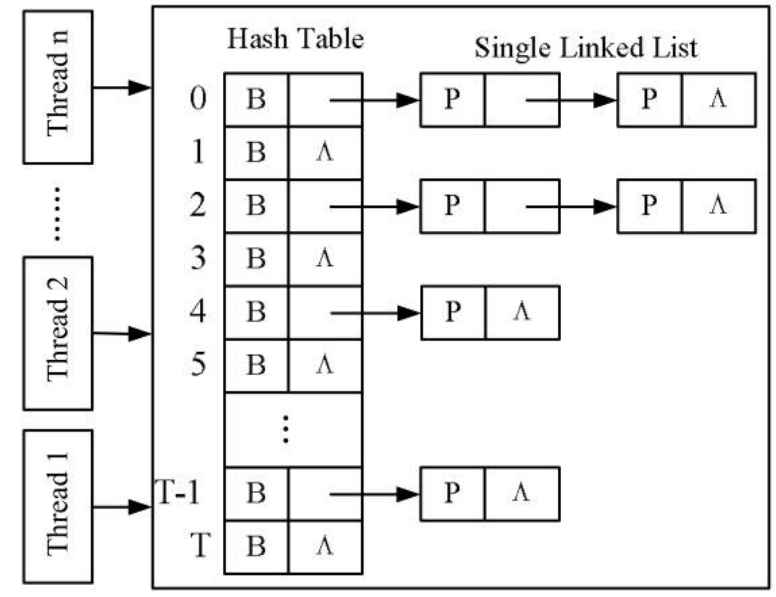

Fig.1 the Basic Structure of Parallel-Hashing

The calculation of each object image, only relates to the object and the $f$ rule, not to other objects. Under multi-core environment, establish multiple calculation threads (in Fig.1) to parallel compute each object image and query in the Hash table. It can further improve the search efficiency. Because multiple threads search possible access to the same array element by multiple threads at the same time. Namely there is an access competition conflict, the key to make parallel hash structure effectively realize is to solve the conflict.

Realize the topological reconstruction method of parallel-hashing. The whole procedure of the method can be divided into three parts: filtering redundancy data, solving concurrent access conflict and the establishment of the topological relation.

This method uses the $n$ triangle number contained in triangular mesh as basic data, with (2) to calculate the $T$ length of vertex table and side table:

$T=2^{\left\lfloor\log _{2} n\right\rfloor}-1$

In the process of merging point table, use three vertex coordinate components with certain magnification to calculate the $K$ critical value of vertex positive integer. Floating-point comparison is converted to integer comparison. The formula of the $K$ critical value and the $f$ Hash function is as follows:

$$
K=\operatorname{int}\left(\frac{\xi \operatorname{int}(|x| S)}{S}+\frac{\rho \operatorname{int}(|y| S)}{S}+\frac{\lambda \operatorname{int}(|z| S)}{S}\right) T
$$

$f(K)=K \bmod T$

Among them, $x, y$, and $z$ are vertex coordinate components. $\xi, \rho$, and $\lambda$ are Hash function coefficients. $S$ is a sensitivity coefficient of Hash function. The mod symbol expresses the remainder computation. The selection of $\xi, \rho, \lambda$ and $S$ is related to data volumes in triangular mesh. Among 
them, as far as possible choose the prime or different irrational number as $\xi$, $\rho$, and $\lambda$, in order to ensure $K$ value the greatest dispersion[9].

It is taken into account that each edge in triangular mesh intersects with the other side only in the two vertexes. Therefore the point coordinate values have spatial uniqueness. Using Hash structure to storage and query side [5], according to edge midpoint coordinates, formula (3) and (4) calculate the $\mathrm{K}$ critical value and the Hash function of edge. In the method of processing edge table hash address conflict, based on the establishment of topological relations between point and boundary, directly use the vertex index value contained in edge to query and merge. It can avoid the error in the half edge matching.

In the parallel hash structure, conflicts in concurrent access are due to multi-threads simultaneously writing order points table, order edges table and the same hash function table address pointing to single list. Although the above method reduces the possibility of concurrent access conflicts, concurrent access conflict is necessary because the hash address conflict is inevitable [2]. Before threads access shared data, lock share datum with lock in operating system [2], in order to prohibit the data to be accessible to other threads. After writing, unlock the shared data, and can be accessed by other threads.

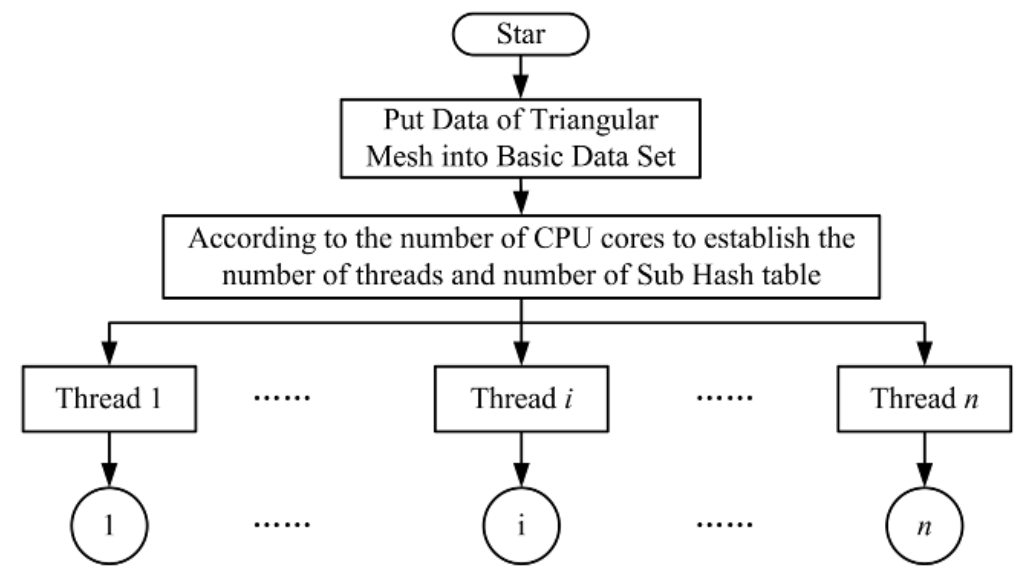

Fig. 2 the Main Flow Chart of Multi-core Program

The topological relation between vertices, edges and triangles is established at the same time with the redundant information filtering and the establishment of non-repeated point table, no repeat edge table and no repeat surface table. Fig.2 and Fig.3 show the final parallel hash topological reconstruction method flowchart. Fig.2 is multi-core parallel main flowchart. The flow of thread $i$ to $n$ is the same as thread 1. Fig.3 is parallel topological reconstruction flowchart of thread 1.

\section{The calculation example and method analysis}

Although the associated reconstruction method uses the relevance of triangular mesh data storage information to improve the computational efficiency, the time complexity of this method is still $O$ $\left(n^{2}+e^{2}\right)$. Where $n$ is the number of vertices in a triangular mesh, $e$ is the number edges [5]. The process is also very difficult to achieve parallelism. Under multi-core environment, make full use of the advantage of Hash algorithm parallelization for triangular mesh topological reconstruction to establish the topological relations between geometric elements, which need to access all the triangles only once. The time complexity is $O(n)$. Therefore it can effectively improve the efficiency of calculation.

This paper designed five kinds of different part structures by using Pro/e software, and saved as the triangular mesh data to different amount of data, as shown in Fig.4. By the correlation method and the present method, the topological information reconstruction was respectively calculated to triangular mesh data are available. Computing environment is as follows: OS is Windows 7 64bit SP1; memory capacity is $8 \mathrm{~GB}$; and CPU is i7 $2.7 \mathrm{GHz}$. Table 1 is the results of using two kinds of method to topological reconstruct for different models. 
The results of example show that the time of topological reconstruction by parallel hashing method is much smaller than by the correlation method, in dual-core mode. By comparing the ratio of the number of handled triangles in triangular mesh and the reconstruction time, it is not difficult to see that the present method basically reflect the linear relationship. But correlation method presents exponential relationship. It consists with previous analysis results. While the ratio of computation time also showed the efficiency advantages of the present method were also more obvious with the increase of the number of triangles.

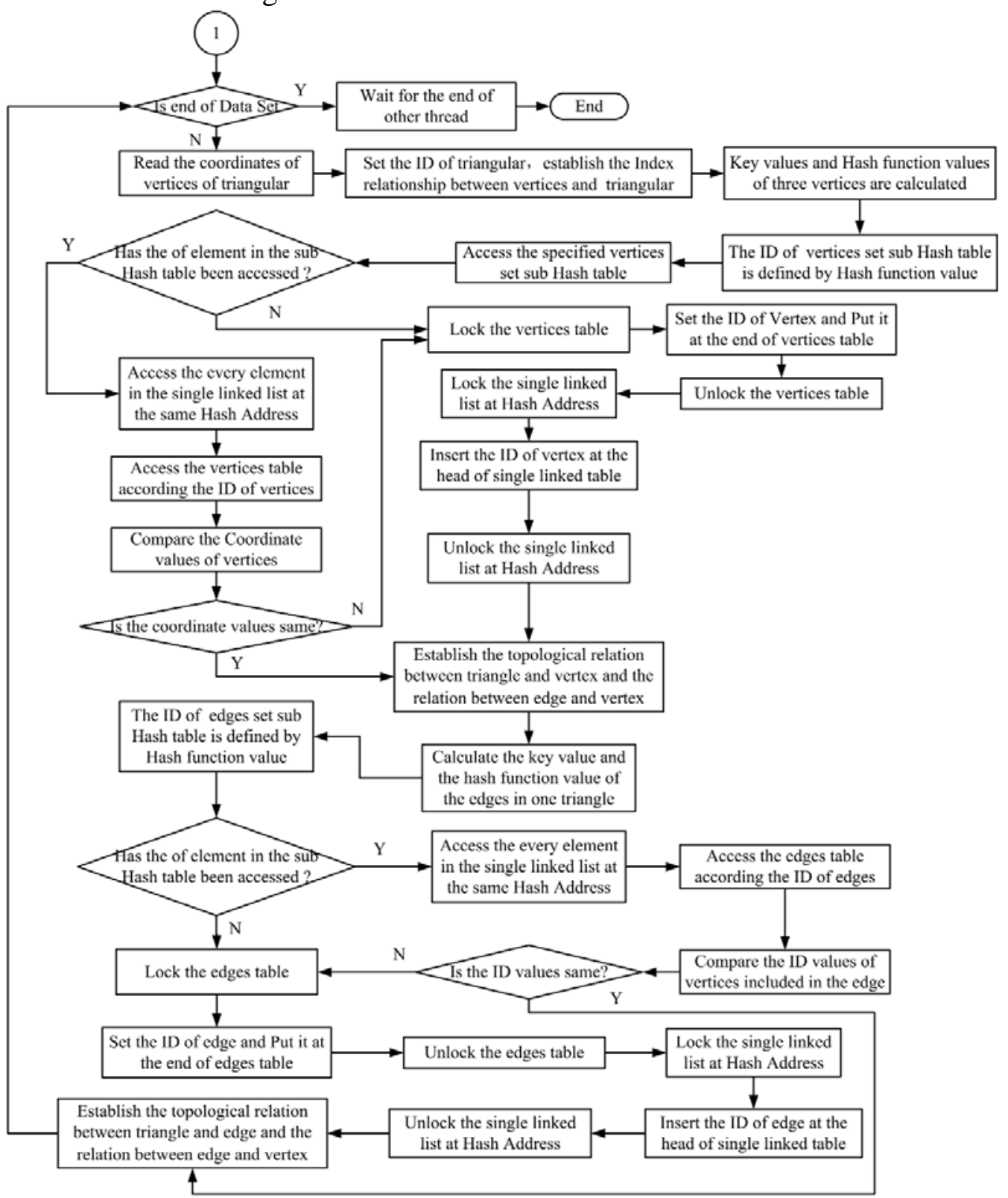

Fig.3 The Main Flow Chart of Parallel Topological Reconstruction Method
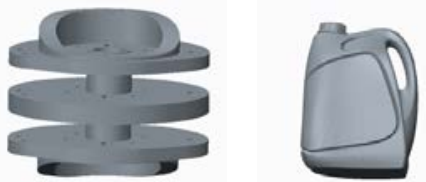

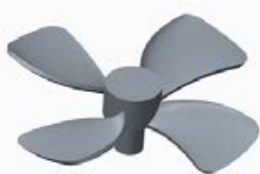

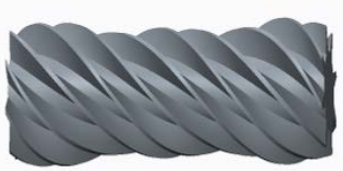

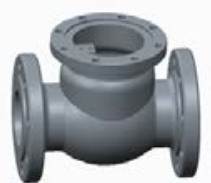
(c) Propeller
(d) Worm
(e) Valve Body
(a) Multi Cam (b) Oil Barrel

Fig. 4 Triangular mesh models used

ed as calculation examples 
Table 1 Results of topological reconstruction for triangular mesh

\begin{tabular}{ccccc|c|c}
\hline $\begin{array}{c}\text { triangular } \\
\text { mesh models }\end{array}$ & $\begin{array}{c}\text { the size of } \\
\text { files(Byte) }\end{array}$ & $\begin{array}{c}\text { the amount } \\
\text { of triangles }\end{array}$ & $\begin{array}{c}\text { the amount } \\
\text { of vertexes }\end{array}$ & $\begin{array}{c}\text { correlation } \\
\text { method(ms) }\end{array}$ & $\begin{array}{c}\text { parallel-Hashing } \\
\text { method(ms) }\end{array}$ & $\begin{array}{c}\text { the ratio of } \\
\text { calculation time }\end{array}$ \\
\hline Multi Cam & $4,130,606$ & 15848 & 7866 & 3105.22 & 115.08 & 26.98 \\
Oil Barrel & $7,643,833$ & 29432 & 14716 & 9841.94 & 222.28 & 44.28 \\
Propeller & $15,759,291$ & 59996 & 30000 & 42039.97 & 487.63 & 86.21 \\
Worm & $29,069,430$ & 113124 & 56564 & 180837.40 & 847.86 & 213.29 \\
Valve Body & $57,935,585$ & 222532 & 111212 & 773673.59 & 1800.23 & 429.76 \\
\hline
\end{tabular}

\section{Conclusion}

This paper, with the effective support of multi-core computing environment to parallel computing, using Hash structure as auxiliary data structure, put forward a new method of the topological reconstruction to triangular mesh model. By improving the Hash function dispersion, this method, effectively reduced the probability of concurrent access conflict, and realized redundant data in triangular mesh model were quickly merge and topological information reconstruction. By using different methods for triangular mesh model on different data, the result of calculation example contrast verified the efficiency and effectiveness of the present method.

\section{Acknowledgements}

This work was financially supported by the Project "The Research on the Method of Getting Manufacturing Quality Features for Complex Curved Surfaces Based on Spatial Data Mining” supported by National Natural Science Foundation of China (No. 51105271), the State Scholarship Fund supported by the China Scholarship Council (No.20140625508), Science and Technology Development Foundation for Colleges and universities of Tianjin (No.20130409). Huijiang Zheng is the correspondent of this paper.

\section{References}

[1] Chen Yongheng, Zuo Xianglin. Parallel Two-Way Enumeration Join Based on Multi-cores Environment [J]. Journal of Jilin University (Science Edition), 2014, 52(1):59-64.

[2] Zhou Weiming. Multicore computing and programming [M]. Wuhan: Huazhong University of Science and Technology Press, 2009.

[3] Szilvási-Nagy M , Mátyási G. Analysis of STL files[J]. Mathematical and Computer Modeling, 2003, 38 (7):945 960.

[4] Zhu Hu, Yang Xiaoguang. A Study on the Interference Detection and Correction in 5-Axis CNC Incremental Forming [J]. Journal of Mechanical Engineering, 2014, 50(7):168-174.

[5] Zheng Huijiang. Research on the Key Technology of the Topological Feature Reconstruction and Sampling Point Distribution in OMV [D]. Tianjin: Tianjin University, 2010.

[6] Qiu Yuanqing, Zhou Huiqun, Zhu Shanshan, Yang Yihu. Topological Reconstruction and Errors Repair of an STL Model Using Hashing [J]. Mechanical Science and Technology, 2009, 28(6):795 798.

[7] Yang Shengyuan, Shu Shi. Fast Reconstruction of STL Surface Meshes Based on Data Correlation [J]. Journal of Computer-Aided Design \& Computer Graphics, 2009, 21(1):67 71.

[8] Yan Weimin, Wu Weimin. Data Structure [M]. Beijing: Qinghua University Press, 1997.

[9] Jan Hradek, Martin Kuchar, Vaclav Skala. Hash functions and triangular mesh reconstruction [J]. Computers \& Geosciences, 2003, 29(6):741-751. 